\title{
In NF1, CFTR, PER3, CARS and SYT7, alternatively included exons show higher conservation of surrounding intron sequences than constitutive exons
}

\author{
Dieter Kaufmann ${ }^{*}{ }^{1}$, Oliver Kenner ${ }^{1}$, Peter Nurnberg ${ }^{2}$, Walther Vogel ${ }^{1}$ and Britta Bartelt ${ }^{1}$ \\ ${ }^{1}$ Department of Human Genetics, University of Ulm, Germany; ${ }^{2}$ Gene Mapping Center, Max Delbrueck Center for \\ Molecular Medicine, Berlin, Germany
}

It is still not fully understood to what extent intronic sequences contribute to the regulation of the different forms of alternative splicing. We are interested in the regulation of alternative cassette exon events, such as exon inclusion and exon skipping. We investigated these events by comparative genomic analysis of human and mouse in five experimentally well-characterized genes, neurofibromatosis 1 (NF1), cystic fibrosis transmembrane conductance regulator (CFTR), period 3 (PER3), cysteinyl-tRNA synthetase (CARS) and synaptotagmin 7 (SYT7). In NF1, high intron identity around the 52 constitutive and four alternatively skipped NF1 exons is restricted to the close vicinity of the exons. In contrast, we found on average high conservation of intron sequences over 300 base pairs up- and downstream of the five alternatively included NF1 exons. The investigation of alternatively included exons in CFTR, PER3, CARS and SYT7 supported this finding. In contrast, the mean intron identities around the alternatively skipped exons in CTFR and NF1 do not differ considerably from those around the constitutive exons. In these genes, the difference in intron conservation could point to a difference between the regulation of alternative exon inclusion and alternative exon skipping or constitutive exon splicing. Additional genome-wide investigations are necessary to elucidate to what extent our finding can be generalized.

European Journal of Human Genetics (2004) 12, 139-149. doi:10.1038/sj.ejhg.5201098

Published online 29 October 2003

Keywords: intron conservation; alternative splicing; alternatively included

Introduction

To generate correct mRNAs in eucaryotes, exons must be identified and joined together precisely and efficiently. In human, this process requires the coordinated action of small nuclear (sn)RNPs interacting with specific DNA

\footnotetext{
*Correspondence: Dr D Kaufmann, Department of Human Genetics, University of Ulm, Albert Einstein Allee 11, Ulm D 89070, Germany. Tel: + 49731500 23419; Fax: + 49731500 23438;

E-mail: dieter.kaufmann@medizin.uni-ulm.de

Online Mendelian Inheritance in Man (OMIM) (http://www3.ncbi.nlm. nih.gov/Omim/); Promoter 2.0 Homepage (http://www.cbs.dtu.dk/ services/Promoter/)

Received 5 March 2003; revised 25 June 2003; accepted 29 August 2003
}

motifs. Well-characterized DNA motifs are the $5^{\prime}$ and the $3^{\prime}$ splice sites, the branch site, and exonic splice enhancers and silencers. ${ }^{1-3}$ Interestingly, also the promoter seems to play a crucial role in alternative splicing. ${ }^{4}$ Several isoforms can be generated by alternative splicing through cassette exon events (ie exon skipping or exon inclusion), exon and intron isoform events (ie use of alternative $3^{\prime}$ or $5^{\prime}$ splice sites) and intron retention. ${ }^{2,3,5}$ We are interested in the regulation of alternative cassette exon events, a cassette exon being one included or excluded from the transcript. The terms exon inclusion and skipping are defined by the absence/presence of the respective alternative exon in the predominant transcript, exons always present in the 
transcript are termed constitutive. ${ }^{5}$ It is still not fully understood to what extent intronic sequences around such exons contribute to the regulation of these forms of alternative splicing. A powerful method to investigate this is the comparative genomic analysis of human and mouse intron sequences. ${ }^{6}$ The availability of both mouse and human genomes allows such investigations on a genomewide level in silico. ${ }^{7}$ However, there are often inconsistent data concerning the classification of alternative cassette exons. Therefore, we chose very well-characterized genes for our investigation. We started with the neurofibromatosis 1 (NF1) gene. NF1 shows numerous constitutive, alternatively included and alternatively skipped exons. NF1 is a tumor suppressor gene and responsible for the human disease NF1 [MIM 162200], which is one of the most common familial tumor syndromes. ${ }^{8,9}$ In human, 61 NF1 exons are known. ${ }^{8-11}$ There are four alternatively included cassette NF1 exons, 9br, 10a-2, 23a and 48a. An additional alternatively included exon, 23b, is expressed in mouse. ${ }^{10}$ The four NF1 exons 4b, 29, 30 and 43 are alternatively skipped in human. ${ }^{11}$ The pattern of expression of the alternative exons is characterized. ${ }^{8-11}$ In addition, there are diverse but rare aberrantly spliced NF1 transcripts. ${ }^{12-15}$ These were not considered alternative in our investigations as they most likely represent splicing noise.

Our investigations demonstrated a high identity of intron sequences over more than 300 base pairs (bp) upand downstream of the alternatively included exons between human and mouse. In contrast, high intron identity around the constitutive and alternatively skipped NF1 exons was restricted to the close vicinity of the exons. Investigation of one of the alternatively included NF1 exons in several species supported the high conservation. We performed similar comparisons with four other experimentally well-characterized genes, the cystic fibrosis transmembrane conductance regulator (CFTR) [MIM 602421], the period 3 (PER3) [MIM 603427], the cysteinyl-tRNA synthetase (CARS) [MIM 123859] and the synaptotagmin 7 (SYT7) [MIM 604146]. These investigations supported the high intron identity around alternatively included exons found in NF1. This finding points to an unknown function of these sequences in the regulation of splicing of alternatively included exons in these genes.

\section{Materials and methods Cell culture}

Primary human fibroblasts, a cell line derived from a rat schwannoma (Rattus norvegicus), fibroblast-like cells derived from mouse (Mus musculus), cat (Felis catus), dog (Canis familiaris), cow (Bos taurus), horse (Equus caballus), sheep (Ovis aries) and griffon vulture (Gyps fulvus) were cultured in DMEM containing $10 \%$ fetal calf serum.
Fibroblasts derived from several primates as the new world monkey Geoffroy's marmoset (Callithrix geoffroyi), the old world monkeys gorilla (Gorilla gorilla) and orang-utan (Pongo pygmaeus), cultured as described above, were kind gifts of W Just, W Krone and W Schemp.

\section{Isolation of cellular RNA and cDNA synthesis}

Total cellular RNA was isolated using the RNeasy spin columns (Qiagen, Hilden, Germany). RNA was quantified by measuring the optical density at $260 \mathrm{~nm}$ and stored at $-70^{\circ} \mathrm{C}$. Total RNA $(1 \mu \mathrm{g})$ was reverse transcribed using the SuperScript ${ }^{\mathrm{TM}}$ First-Strand Synthesis system and random hexamers (Invitrogen Life Technologies, Carlsbad, CA, USA). cDNA derived from Fugu rubripes was a kind gift of H Kehrer-Sawatzki.

\section{Expression analysis of NF1 10a-2}

Human cDNA was amplified using the primers $\mathrm{Nf} 9 \mathrm{H}$ and Nf10bR (primer sequences see Table 1) resulting in a wildtype amplification product of $306 \mathrm{bp}$ representing sequences of NF1 exons 9, 10a and 10b. The amplification conditions in the thermal cycler GeneAmp 9600 (PerkinElmer, Boston, MA, USA) were denaturation at $94^{\circ} \mathrm{C}$ for 2 min followed by 30 cycles of $93^{\circ} \mathrm{C}$ for $30 \mathrm{~s}, 49^{\circ} \mathrm{C}$ for $30 \mathrm{~s}$ and $72^{\circ} \mathrm{C}$ for $30 \mathrm{~s}$ and a final extension step at $72^{\circ} \mathrm{C}$ for $10 \mathrm{~min}$. The reamplification was performed as described. ${ }^{9}$ In addition, cDNA was amplified using the NF1 10a-2specific primer Nf10a2H and primer Nf10bR, annealing at $46^{\circ} \mathrm{C}$, 40 cycles, resulting in a PCR product of $157 \mathrm{bp}$. cDNA from different species was amplified using the same primer pairs as for human cDNA. Fugu rubripes cDNA was tested for the expression of NF1 10a-2 with primer pair Fugu-Nf7-H/ Fugu-Nf10b-R, annealing at $61^{\circ} \mathrm{C}$, yielding a wild-type product of $560 \mathrm{bp}$. The region where the NF1 10a-2 product was expected was gel extracted and reamplified with the same primer pair. Alternatively, a reamplification with a specific forward primer Fugu-Nf10a-2-H and Fugu-Nf10b-R, annealing at $65^{\circ} \mathrm{C}$, was performed, resulting in a product of $198 \mathrm{bp}$.

DNA isolation and amplification from several species To analyze the NF1 intron 10a sequence of various species gDNA was isolated from cells either with the DNeasy Kit (Qiagen, Hilden, Germany) or with the salting-out method. PCR products were generated with the primer pairs in Table 1 using system 1 of the Expand Long Template PCR System (Roche Diagnostics, Mannheim, Germany). PCR conditions in a Perkin-Elmer GeneAmp 9700 were: 2 min at $92^{\circ} \mathrm{C}$, then 10 cycles of $10 \mathrm{~s}, 92^{\circ} \mathrm{C}, 30 \mathrm{~s}$ annealing (60$65^{\circ} \mathrm{C}$ ), $8 \mathrm{~min}, 68^{\circ} \mathrm{C}$, then 20 cycles of $10 \mathrm{~s}, 92^{\circ} \mathrm{C}, 30 \mathrm{~s}$ annealing, $10 \mathrm{~min}, 68^{\circ} \mathrm{C}$ and a final elongation of $10 \mathrm{~min}$ at $68^{\circ} \mathrm{C}$. Primer pairs for cow and sheep DNA In10aH/In10aR, Cow-In10a-H/In10aR and Cow2-In10a-H/In10aR. Annealing was $65^{\circ} \mathrm{C}$ for all three pairs. Primer pairs for horse DNA: 
Table 1 Primers used for the amplification of sequences containing NF1 exon 10a-2 and of NF1 intron 10a in several species

\begin{tabular}{|c|c|c|}
\hline Species & Name & Sequence \\
\hline Cat & $\begin{array}{l}\text { Cat10aH } \\
\text { Cat10aR } \\
\text { Cat3-In10a-H } \\
\text { Cat3-In10a-H } \\
\text { Cat3-In10a-H } \\
\text { Cat-In10a-H }\end{array}$ & $\begin{array}{l}\text { 5'-CTA GCT AAT GGT GTT TGT TCT TCA-3' } \\
\text { 5'-TCA ATT GGT TCC ATA CGA GTT TTA-3' } \\
\text { 5'-GGC GCA CAC CCA GCA ATA C-3' } \\
\text { 5'-GGC GCA CAC CCA GCA ATA C-3' } \\
\text { 5'-GCG CAC ACC CAG CAA TAC-3' } \\
\text { 5'-CAT TGA TTG GTG GTG CTT TGT CTT CT-3' }\end{array}$ \\
\hline Cow/sheep & $\begin{array}{l}\text { Cow-In10a-H } \\
\text { Cow2-In10a-H }\end{array}$ & $\begin{array}{l}\text { 5'-CAC TTT TCT CAC ACA TAA ACA TTG GGA-3' } \\
5^{\prime} \text {-AGC TTT CTT TGT TCT ATC AGT GTT CTT CT-3' }\end{array}$ \\
\hline Dog & Dog2-In10a-R & 5'-CAA CAG CAG CCA ATA AGA ACA GAA-3' \\
\hline Fugu & $\begin{array}{l}\text { Fugu-Nf7-H } \\
\text { Fugu-Nf10b-R }\end{array}$ & $\begin{array}{l}\text { 5'-CGC CGC CAT CGC CTG TGT C-3' } \\
5^{\prime} \text {-TGG GGT CTG CGT GGA TGA GTT TGA-3' }\end{array}$ \\
\hline Horse & $\begin{array}{l}\text { Horse3-In10a-H } \\
\text { Horse3-In10a-R }\end{array}$ & $\begin{array}{l}\text { 5'-TAG GCT CTT TGA TAT TGA AGT TTG TGT TT-3' } \\
5^{\prime} \text {-CCC TTT GTA AGA ATC AGA CAT CAG AAC T-3' }\end{array}$ \\
\hline Mouse & $\begin{array}{l}\text { Mouse-In10a-R } \\
\text { Mouse-In10a-H }\end{array}$ & $\begin{array}{l}\text { 5'-CAT TTG TAG CTC CTT GTC TCT AGG TCT-3' } \\
5^{\prime} \text {-AGA TTG TGC CCA TGG TTT CCT TAC TC-3' }\end{array}$ \\
\hline Vulture & $\begin{array}{l}\text { Vulture-In10a-H } \\
\text { Vulture-In10a-R } \\
\text { Vulture2-In10a-H }\end{array}$ & $\begin{array}{l}\text { 5'-GGC CCA AGA TCG ATG CTG TTT ACT G-3' } \\
5^{\prime} \text {-CAG CAT GGA TCA GTT TTA CCA AGG ATA A-3' } \\
\text { 5'-TCA TTT GTC TTG CTT GCT TTC TGT TGA G-3' }\end{array}$ \\
\hline Human & $\begin{array}{l}\text { Nf10aH } \\
\text { Nf10a-nested-H } \\
\text { Nf9H } \\
\text { Nf10bR } \\
\text { In10aH } \\
\text { In10aR }\end{array}$ & $\begin{array}{l}\text { 5'-CAT TGG ATT GGT GGC CTA AGA-3' } \\
\text { 5'-TTG ATG CTG TGT ATT GTC ACT C-3' } \\
\text { 5'-CTG GCT CAG AAT TCA CCT TCT-3' } \\
\text { 5'-TTA GTT TCA CCA TGG ACA AGA G-3' } \\
\text { 5'-TTG GAT TGG TGG CCT AAG ATT GAT GC-3' } \\
\text { 5'-TAC TTA TAG CTT CTT TCT CCA GGT CT-3' }\end{array}$ \\
\hline
\end{tabular}

In10aH/In10aR, annealing at $65^{\circ} \mathrm{C}$ and Horse3-In10a-H/ Horse3-In10a-R, annealing at $63^{\circ} \mathrm{C}$. Primer pairs for mouse DNA: In10aH/Mouse-In10a-R, annealing at $65^{\circ} \mathrm{C}$ and Mouse-In10a-H/Mouse-In10a-R, annealing at $62^{\circ} \mathrm{C}$. Primer pairs for cat DNA: Cat-In10a-H/In10aR, annealing at $63^{\circ} \mathrm{C}$. Primer pairs for dog DNA: Cat-In10a-H/In10aR, annealing at $60^{\circ} \mathrm{C}$. Primer pairs for vulture DNA: GW-In10a-H/GWIn10a-R and GW2-In10a-H/GW-In10a-R, both annealing at $62^{\circ} \mathrm{C}$. PCR products of cat and dog DNA were also generated with Taq DNA polymerase (Amersham Pharmacia Biotech, Uppsala, Sweden) and 30 cycles of $30 \mathrm{~s}$ at $92^{\circ} \mathrm{C}$, $30 \mathrm{~s}$ annealing and $30 \mathrm{~s}$ at $72^{\circ} \mathrm{C}$. Primer pairs for cat were: (1) Nf10aH/Nf10a2R, annealing at $49^{\circ} \mathrm{C}$; (2) Nf10a-nested$\mathrm{H} / \mathrm{Nf10a} 2 \mathrm{R}$, annealing at $52^{\circ} \mathrm{C}$ and (3) Cat10aH/Cat10aR, annealing at $55^{\circ} \mathrm{C}$. Primer pair for dog was: Cat3-In10a-H/ Dog2-In10a-R, annealing at $54^{\circ} \mathrm{C}$. Intron 9 of the griffon vulture NF1 gene was amplified with Taq DNA polymerase and primer pair GW-In9-H/GW-In9-R and GW-In9-H3/ GW-In9-R, annealing at $65^{\circ} \mathrm{C}$. All PCR products were purified by gel extraction or PCR product purification with the GFX kit (Amersham Pharmacia Biotech, Uppsala, Sweden) and sequenced on an ABI 373A automated sequencer (PE Applied Biosystems, Foster City, CA, USA). PCR products from gDNA were sequenced using only the respective forward primers.
Search and criteria for the genes investigated GenBank (http://www.ncbl.nlm.nih.gov/Genbank/ GenbankSearch.html), the AltExtron database (http:// www.ebi.ac.uk/asd/altextron/access.html) and PubMed (http://www.ncbi. nlm.nih.gov/entrez/query.fcgi1) were searched for genes with alternatively included cassette exons conserved between human and mouse and ideally surrounded by several hundred base pairs of intronic sequences. Of these, the majority of the genomic sequence of human and mouse or rat had to be available and they should contain at least 10 exons and sufficiently sized introns to ensure enough data for statistical analysis.

Comparative sequence analysis of intron conservation Alignment of the NF1 intron 10a sequences of various species to the human sequence was carried out using the BestFit agorithm (GCG, Accelrys, Burlington, MA, USA) with the most stringent gap creation and gap extension penalties yielding a complete alignment. The rat intron 10a sequence was obtained from the Rat Genome Project (http://www.hgsc.bcm.tmc.edu/projects/rat/). For the calculation of mean intron identities around all constitutive NF1 exons except exon 1, the human and mouse NF1 intron sequences together with the flanking exons were aligned with BestFit. Alignment was carried out in sections 
of one intron plus the surrounding exons. The NF1 sequences of human and mouse were obtained from GenBank. Nf1 exon 1 and intron 1 have not yet been sequenced in mouse. The identities of 50 nucleotides (nt) windows $350 \mathrm{bp}$ up- and downstream of the respective exons were calculated. Statistical analysis of the difference between the graphs of the mean values of alternatively included, alternatively skipped and constitutively spliced exons was performed with the paired $t$-test. The alignments of the NF1 intron 10a sequences were used to calculate the identities for $50 \mathrm{nt}$ windows $600 \mathrm{bp}$ up- and downstream of exon 10a-2, which lies $764 \mathrm{bp}$ downstream of exon 10a in the $8 \mathrm{~kb}$ sized intron $10 \mathrm{a}$. To generate random sequences with the same dinucleotide frequency as the NF1 gene, the complete human genomic NF1 sequence was randomized with Shuffle from the GCG package under the preservation of the dinucleotide frequency. Five sequences of $1250 \mathrm{nt}$ each were then taken from the resulting sequence and compared to the human sequence of the five alternatively included and five randomly chosen constitutive NF1 exons and $600 \mathrm{bp}$ of intron sequence up- and downstream as described above. The identities for $50 \mathrm{nt}$ windows were calculated and averaged for all alternatively included exons $\left(n_{\text {windows }}=683\right)$ and the five constitutive exons $\left(n_{\text {windows }}=725\right)$. Likewise, five random sequences of $1250 \mathrm{nt}$ each, representing each base equally, were compared to the same NF1 sequences described above. Again, the identities for $50 \mathrm{nt}$ windows were calculated and averaged for all alternatively included exons $\left(n_{\text {windows }}=649\right)$ and the five constitutive exons $\left(n_{\text {windows }}=617\right)$. Alignment of the cat and mouse CFTR sequences to the human sequence was carried out as described for NF1 except for very large introns or introns extremely differing in size where only smaller parts of the sequences were aligned. Multiple alignment of the previously unknown CFTR intron 10 element of various species was carried out with Dialign (http://www. genomatix.de/cgi-bin/diallgn/dialign.pl). PER3 and WT1 sequences were also obtained from GenBank, CARS and SYT7 sequences from the Celera Discovery System and the alignment carried out as described for NF1.

\section{Search for intron sequence elements}

In all, 600 bp up- and downstream of all alternative NF1 and CFTR exons investigated were examined for several known intronic sequence elements with FindPatterns or BestFit of the GCG package. We tested for the following pattern sequences: Nova-1 binding sequence; ${ }^{16}$ (A/U)GGG repeats; ${ }^{17}$ PTB binding site; ${ }^{18}$ SF1 binding site; ${ }^{19}$ UGCAUG repeats; ${ }^{20}$ hnRNP A1 binding site; ${ }^{21}$ multiple copies of UGC; ${ }^{22}$ GAR repeats; ${ }^{23}$ CE4m repressor; ${ }^{24}$ IAS 2 and IAS $3 ;^{25}$ $5^{\prime}$ splice site consensus; ${ }^{26}$ downstream control sequence; ${ }^{27}$ conserved intronic element; ${ }^{28}$ and muscle-specific enhancer. $^{29}$ The conserved intron sequences surrounding the alternative exons were examined for RNA polymerase II promoter sequences with CorePromoter (http://argon.cshl.org/genefinder/CPROMOTER/index.htm), ${ }^{30}$ Neural Network Promoter Prediction (http://www.fruitfly.org/ seq_tools/promoter.html) and Promoter 2.0 (http:// www.cbs.dtu.dk/services/Promoter/). ${ }^{31}$ Search for RNA polymerase III promoter sequences was carried out with FindPatterns, including the $\mathrm{A}$ and $\mathrm{B}$ box consensus sequences of the type II promoter, the TATA box, PSE, DSE and Sp1 consensus ${ }^{32}$ elements of the type III promoter. Sequences characteristic of snoRNAs, such as the C, D and $\mathrm{H}$ box consensus sequences ${ }^{33}$ were also searched for with FindPatterns. The intron sequences containing alternative exons were searched for open reading frames in sense and antisense orientation with the algorithm Frames of the GCG package.

\section{Accession numbers}

http://www.ncbi.nlm.nih.gov/entrez/query.fcgi?db = Nucleotide, NCBI GenBank Entrez Nucleotide (for NF1 intron $10 \mathrm{a}$ sequenced by us [AF457133] to [AF457138]; for human NF1 [NT_010799]; for mouse Nf1 exons 2-27b [AL591126]; for mouse Nf1 exons 28-49 [AC008161]; for human CFTR [AC000111] and [AC000061]; for cat CFTR [AC091436] and [AC091382]; for mouse Cftr [AF162137]; for Pan troglodytes CFTR [AC087834]; for C. familiaris CFTR [AC091119]; for $R$. norvegicus CFTR [AC091268]; for B. taurus CFTR [AC089993]; for Sus scrofa CFTR [AC092478]; for human PER3 [Z98884]; for mouse Per3 [AL607143]; for human WT1 [AL049692]; for mouse Wt1 [AL5125841]). https://industry.ebi.ac.uk/altExtron/, AltExtron Database Project (for alternative PER3 exon [IDB61710]). http://www.hgsc.bcm.tmc.edu/projects/rat/, Rat Genome Project (for rat NF1 intron 10a [project_gjdy_BT]). http://www.celeradiscoverysystem.com/index. cfm, Celera Discovery System (for human CARS [hCG16441]; for mouse CARS [mCG15274]; for human SYT7 [hCG40863]; for mouse SYT7 [mCG1938]).

\section{Results \\ Intron sequence conservation between human and mouse is higher around alternatively included than constitutive NF1 exons}

We compared NF1 sequences from human and mouse using the BestFit algorithm. First, we determined the basic identity generated by this algorithm. Random sequences representing the same dinucleotide frequency as the NF1 gene were generated. We aligned these random sequences to the human intron sequences around the five randomly chosen constitutively spliced NF1 exons $2,12 \mathrm{~b}, 31,39$ and 41 , and around the five alternatively included NF1 exons 9br, 10a-2, 23a, 23b and 48a. The investigation yielded an average identity of $46.5 \pm 0.28 \%$ (SEM) per $50 \mathrm{nt}$ window for the constitutively spliced exons and $46.2 \pm 0.31$ (SEM) for the alternatively included exons. We considered this 


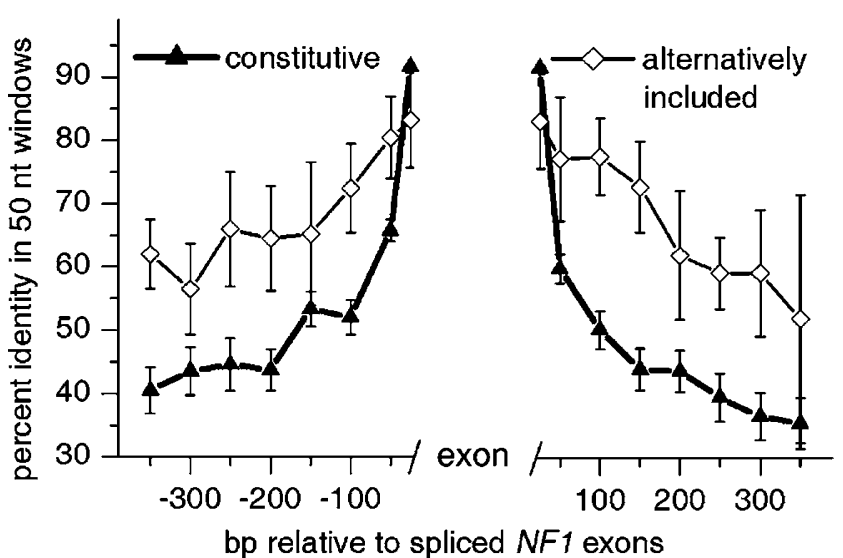

Figure 1 Comparison of the intron sequences around alternatively included and constitutive NF1 exons between human and mouse. Mean identities of $50 \mathrm{nt}$ windows $350 \mathrm{bp}$ up- and downstream of 52 constitutively spliced NF1 exons (black triangles, error bars: SEM) are lower than those of all five alternatively included NF1 exons (white rhombuses, error bars: SEM).

identity the basic identity for our investigations. Intron alignment of 52 constitutive NF1 exons of human and the corresponding exons of mouse yielded on average high identity $(>60 \%$ ) only in close vicinity to the exons. The identity declined to values nearing the basic identity at a distance of $100 \mathrm{bp}$ from the exon-intron boundary (Figure 1). In contrast to the constitutively spliced exons, the mean identity of the intron sequences surrounding the alternatively included NF1 exons is higher for every $50 \mathrm{nt}$ window of the $350 \mathrm{bp}$ (Figure 1). The mean values of the two graphs differ significantly (paired $t$-test, $P<0.001$ ). The individual alternatively included NF1 exons show differences in intron conservation (data not shown). We found the most extended region of high identity around NF1 exon $23 \mathrm{a}$, followed by exons $10 \mathrm{a}-2,23 \mathrm{~b}$ and $9 \mathrm{br}$. The intron sequences around the poorly conserved NF1 exon 48a are not remarkably identical except for a sequence stretch $100 \mathrm{bp}$ upstream of exon 48a, which may represent an unknown essential element (see below and Table 2). Even if the exceptionally conserved sequences surrounding exon 23a are not considered, the mean values of the intron sequences surrounding the remaining four exons differ significantly from the mean values of the introns of the constitutively spliced exons (paired $t$-test, $P<0.001$ ). The high intron homology around exon $23 \mathrm{~b}$ is remarkable because expression of this exon is found in mouse but not yet in human. ${ }^{8,15}$ We suggest that it is expressed in specific situations not yet investigated in human. The investigation of the human sequence of exon $23 \mathrm{~b}$ and its surrounding intronic sequences showed that the splice sites are almost $100 \%$ identical. Only the reading frame is altered due to a $1 \mathrm{bp}$ deletion in the human sequence, which would lead to an earlier premature stop codon as in mouse.

\section{High conservation of intron sequences surrounding the alternatively included NF1 exon 10a-2 in several species}

The observation of the high intron homology around the NF1 exon 10a-2 between human and mouse is also remarkable because we found the expression of this exon to be lost in mouse. The invariant $\mathrm{T}$ of the $5^{\prime}$ splice site consensus is altered to $G$, the putative branch point shows a single base exchange and the mouse exon 10a-2 homologous sequence comprising $40 \mathrm{bp}$ would lead to a premature stop codon formed by the last base of exon 10a2 and the first two bases of exon 10b (Figure 2). The high intron homology without the expression of the exon could on the one hand indicate that the expression was lost only recently in mouse leaving too little time for the accumulation of mutations in the intron or on the other hand that an active constraint independent of exon 10a-2 prevents mutation of the intronic sequences. To elucidate this, we investigated several species with different times of divergence (eg vulture-human 310 million years ago, mouse, rat, dog, cat, horse-human 90 million years $\mathrm{ago}^{34}$ ) for exon 10a-2 presence, expression and intron identity (Figures 2 and 3). We detected the first reliable expression of exon 10a-2 in bird (griffon vulture). The homology of the bird to the human 10a-2 sequence is high as shown by sequencing of cDNA and gDNA (Figure 2). In intron 10a of the NF1 gene of the pufferfish Fugu rubripes, we identified a nonexpressed $62 \mathrm{bp}$ sequence with $50 \%$ identity to the

Table 2 Previously unknown conserved sequence elements observed in the intron sequences surrounding alternatively included exons

\begin{tabular}{|c|c|c|c|}
\hline Exon & Location relative to exon & Sequence & Identity to human \\
\hline CFTR 10b & $-11 b p$ & $\begin{array}{l}\text { GCA CAA CAT ATT TCA CAT AGT TTT } \\
\text { CTG ATT TCA GT }\end{array}$ & $\begin{array}{l}\text { Old world monkeys: } 100 \% \text {; Mouse, } \\
\text { rat, cat: } 100 \% \text {; cow: } 94.3 \% \text { dog: } \\
\text { 91.4\%; pig: } 88 \%\end{array}$ \\
\hline
\end{tabular}



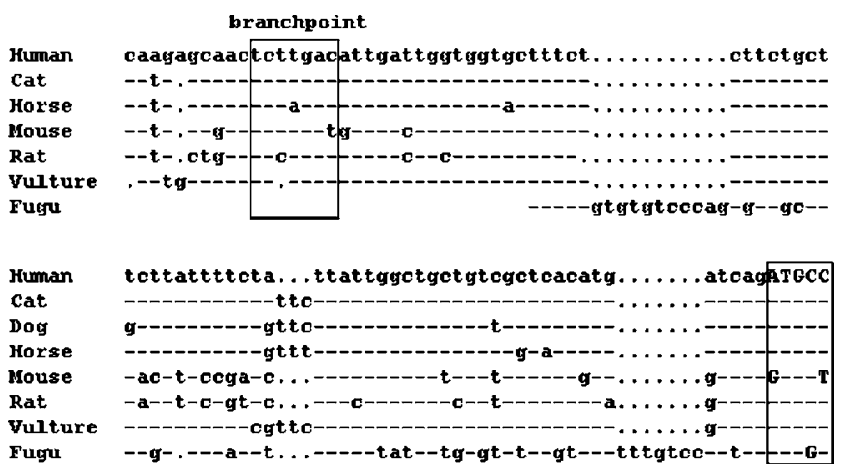

Fugu

exon 10a-2
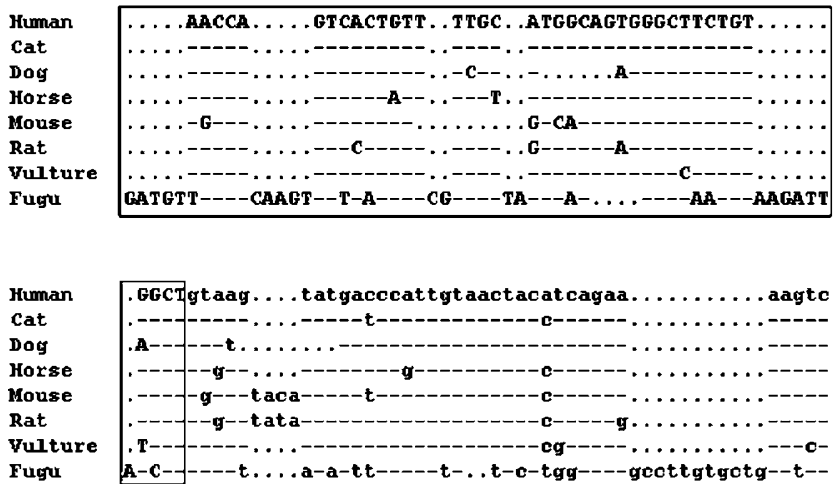

Figure 2 Sequence homology between NF1 exon 10a-2 of human and cat, dog, horse, mouse, rat, vulture and Fugu. Nucleic acid sequence alignment of NF1 10a-2 and surrounding intron sequences between several species. The human sequence is shown in capital letters. A match to the human sequence is represented by a dash, a gap by dots. The exon 10a- 2 homologous sequence and the putative branch point are highlighted boxes.

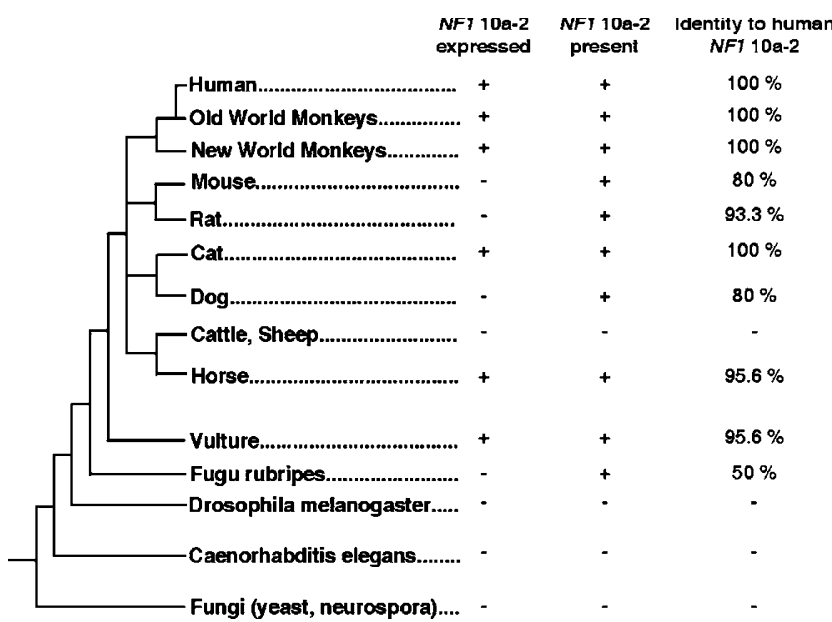

Figure 3 Phylogenetic tree showing the relationship between the species investigated for alternatively included NF1 exon 10a-2. For each species the information whether exon 10a-2 is expressed and/or present in the genomic DNA sequence is shown, and, if present, its identity to the human sequence. human NF1 10a-2 (Figure 2). We also found highly conserved exon 10a-2 homologous sequences in horse, $\mathrm{dog}$, cat, rat and all primates investigated. Of these species, $10 \mathrm{a}-2$ is expressed in horse, cat and primates. We did not find 10a-2 expression in rat, although the splice sites are both intact, the homology to the human exon 10a- 2 is very high (93.3\%) and it does not lead to an alteration of the reading frame (Figure 2). Only the putative branch point shows a $1 \mathrm{bp}$ alteration different from that seen in mouse. It seems therefore likely that the rat exon $10 \mathrm{a}-2$ is expressed but in cell types not investigated by us. As even rat does not harbor the same alterations as mouse and the divergence of rat and mouse is estimated at 40 million years ago, ${ }^{34}$ the loss of exon 10a-2 expression is clearly a very recent event in mouse. We therefore assume that the time span since this loss was insufficient to greatly alter the surrounding intronic sequences. The alignment of the introns surrounding exon 10a-2 of those species with this exon to the human introns revealed a very high identity over several hundred base pairs for cat and horse (Figures 2 and 4). Also rat and dog show remarkably high intron identity, although not expressing exon 10a-2. In vulture, high identity is restricted to about $100 \mathrm{bp}$ up- and downstream
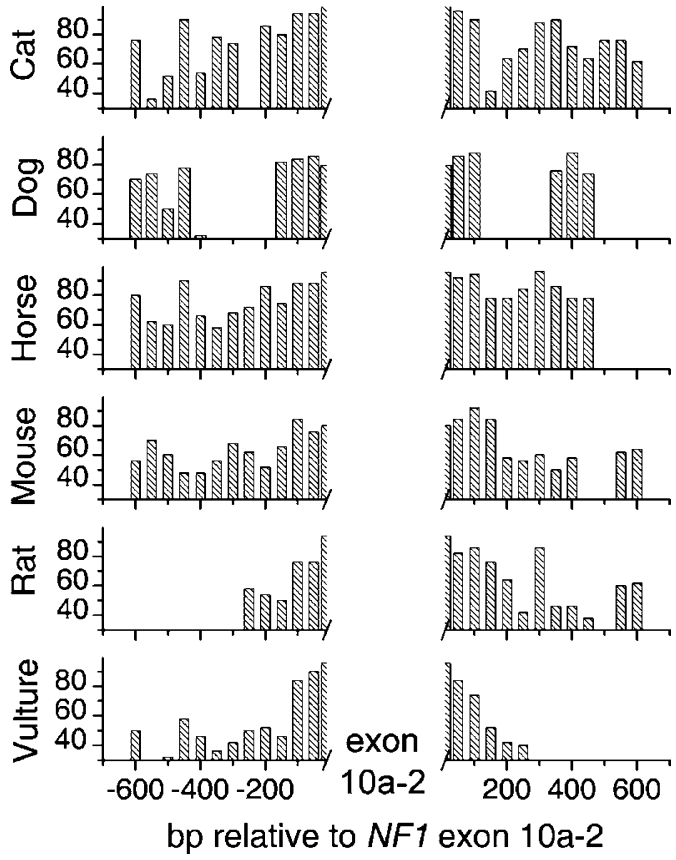

Figure 4 Comparison of NF1 exon 10a-2 and surrounding intron sequences between human and several species. The identities of $50 \mathrm{nt}$ windows of alignments between the human intron 10a sequence and that of several other species (cat, dog, horse, mouse, rat and vulture) were calculated for 600 bp up- and downstream of exon 10a-2. The identities are displayed as columns. Gaps in the alignment due to insertions/deletions are shown as an identity of zero. 
of the exon. Taken together, we could confirm the high conservation of the intron sequences surrounding exon 10a- 2 observed in mouse in several other species and we could show that the loss of exon 10a- 2 expression is a very recent event in mouse.

Higher conservation of intron sequences surrounding alternatively included CFTR, PER3, CARS and SYT7 exons

To investigate if high intron identity around alternatively included exons is a more general phenomenon, we examined four other very well-characterized genes fitting our criteria (see Materials and methods), the CFTR, the PER3, the CARS with one alternatively included exon each and the SYT7 with five alternatively included exons. The intron identities around the conserved alternatively included CFTR exon $10 b^{35}$ are higher than that of the average intron identities around the 16 constitutively spliced CFTR exons between human and cat (Figure 5a) and for the most part also between human and mouse (data not shown). PER3 comprises 21 exons in human homologous to exons $2-22$ of the mouse mPer $3^{36}$ and contains one alternatively included exon, which we have designated $2 \mathrm{a}$. The comparison revealed intron identities higher than the average identities around the constitutive exons downstream of exon $2 \mathrm{a}$, while upstream only the identity of the first $50 \mathrm{nt}$ window is higher than the average (Figure $5 \mathrm{~b}$ ). CARS is composed of 23 exons in human and mouse, 20 of which are constitutively spliced. ${ }^{37}$ The alternatively included exon 2 shows as the PER3 exon mainly intron identities higher than the average identities around the constitutive exons downstream of the exon (Figure 5c). SYT7 comprises 14 exons, five of which, exons $4-8$, are

Figure 5 Intron conservation around alternatively included CFTR, PER3, CARS and SYT7 exons in comparison to the intron conservation of the respective constitutively spliced exons. (a) Average intron identities of all constitutive CFTR exons (black triangles) in comparison to the intron identities around CFTR exon 10b (columns) between human and cat. The generally higher similarity of not transcribed sequences is surprising, because the evolutionary distance of these species is in the same order of magnitude as that of man and mouse. It somehow parallels the similarity of the karyotypes, which has been found to be close to the ancestral mammalian chromosome arrangement in both cases. ${ }^{49}$ (b) Average intron identities of all constitutive PER3 exons (black triangles) in comparison to the intron identities around PER3 exon 2a (columns) between human and mouse. (c) Average intron identities of all constitutive CARS exons (black triangles) in comparison to the intron identities around CARS exon 2 (columns) between human and mouse. (d) The average intron identities of the nine constitutive SYT7 exons (black triangles) in comparison to the average intron identities around the five alternatively included SYT7 exons (white rhombuses) between human and mouse. alternatively included. ${ }^{38}$ The average intron identities around all alternatively included exons are clearly higher than the average identities around the constitutive exons
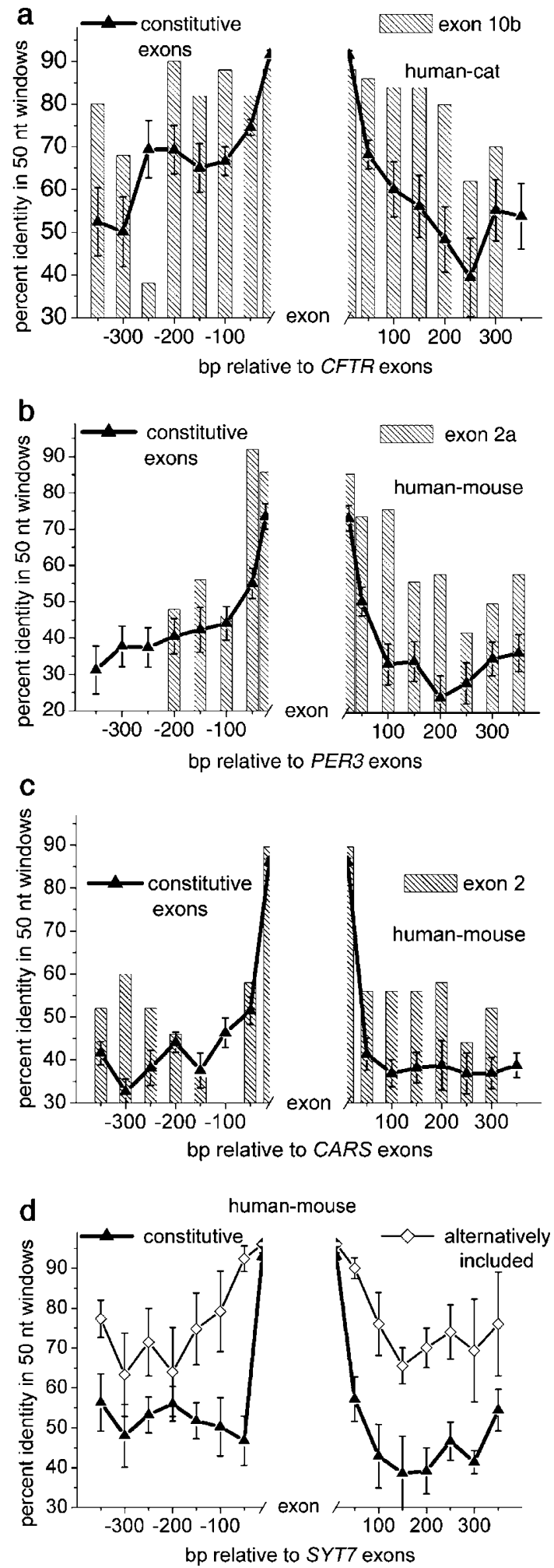
(Figure 5d). Taken together, this points to high intron identity around alternatively included exons being a more general phenomenon.

\section{Restricted intron conservation around alternatively skipped NF1 and CFTR exons}

Then, we investigated the introns surrounding alternatively skipped cassette exons. The NF1 exons 4b, 29, 30 and $43^{11,12}$ and the CFTR exons $2,3,4,5,9,11,12$ and $14 \mathrm{a}^{39}$ are alternatively skipped. Interestingly, the mean intron identities around these skipped exons do not differ considerably from those around the constitutive exons (Figures $6 \mathrm{a}$ and $\mathrm{b}$ ). This shows a difference in the conservation of surrounding intron sequences between alternatively included and alternatively skipped exons. Our finding is supported by the investigation of the Wilms' tumor 1 gene (WT1) consisting of eight constitutive exons and one alternatively skipped exon. ${ }^{40}$ The mean intron identities around the skipped exon 5 do not differ from those around the constitutive exons (data not shown).

Other differences between alternatively included and skipped exons

Recently, other differences between alternatively included and skipped cassette exons have been reported as smaller size of the included exons. ${ }^{5}$ The five alternatively included NF1 exons are indeed much smaller (mean $46.4 \mathrm{bp}$ ) than the four skipped NF1 exons (mean $193 \mathrm{bp}$ ). The one alternatively included CFTR exon (119bp), however, is not that much shorter than the average of the eight skipped exons (127.5 bp). Small size does not seem to correlate with high intron identity, as in NF1 the identities around the three smallest constitutive small exons 4c (68bp), 35 (62 bp) and 47 (47bp) do on average not differ from those found for the constitutive exons (data not shown). It was also shown that the donor splice sites of included cassette exons show a stronger consensus to A at position +3 (A3) than alternatively skipped and constitutive exons. ${ }^{1,5}$ However, we see no differences between the six included (all A3) and 12 skipped (11 A3, 1 G3) alternative exons investigated in NF1 and CFTR.

Known elements and unknown conserved sequences in the introns surrounding alternatively included NF1 and CFTR exons

The high intron identity found around alternatively included exons raised the question about the function of these sequences. The investigation of the introns around the alternatively included NF1 and CFTR exons for several known intronic splice elements mostly present in several copies revealed several matches conserved between human and mouse but no correlation with the conserved sequences. Also, the investigation of the introns showed
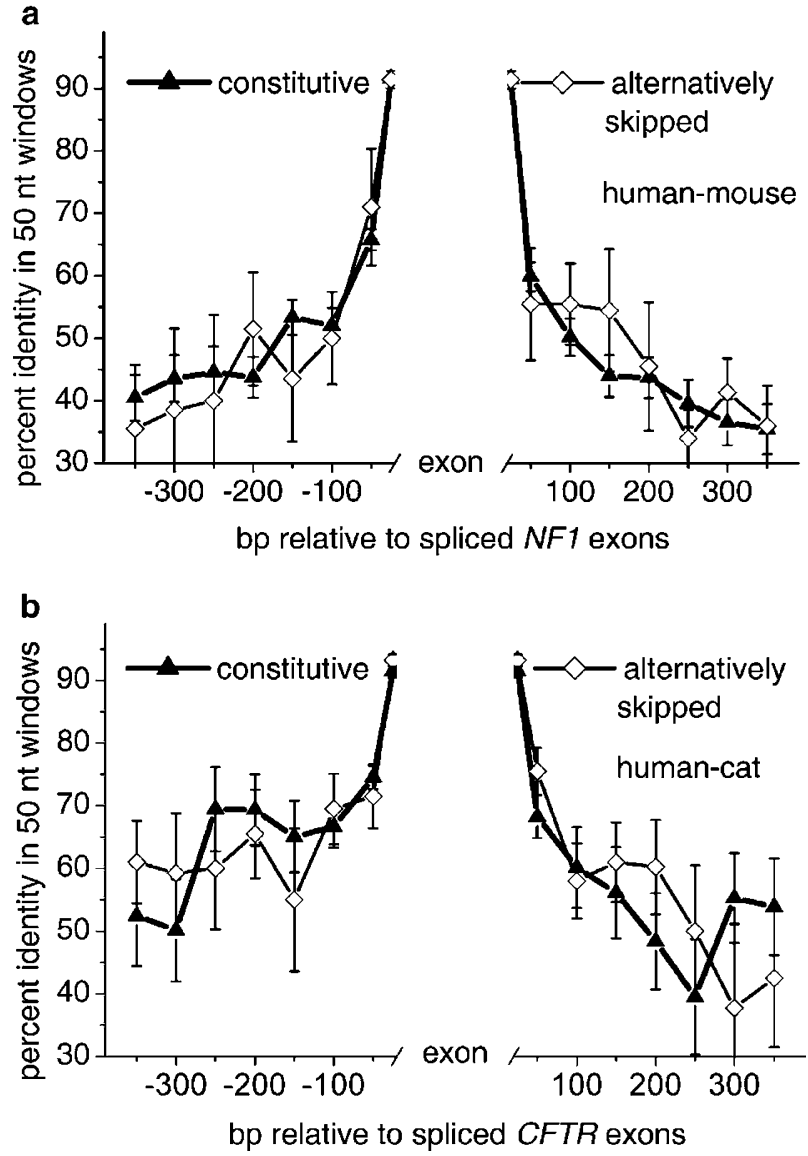

Figure 6 The intron conservation around alternatively skipped NF1 and CFTR exons is not higher than that around the constitutive exons. (a) The mean identities of $50 \mathrm{nt}$ windows $350 \mathrm{bp}$ up- and downstream of all four alternatively skipped NF1 exons (white rhombuses; error bars: SEM) between human and mouse do not differ significantly (paired $t$-test: $P>0.9999)$ from those of 52 constitutively spliced NF1 exons (black triangles; error bars: SEM). (b) The mean identities of $50 \mathrm{nt}$ windows $350 \mathrm{bp}$ up- and downstream of all eight alternatively skipped CFTR exons (white rhombuses; error bars: SEM) between human and cat and of 16 constitutively spliced CFTR exons (black triangles; error bars: SEM) also do not differ significantly (paired $t$ test: $P>0.939)$.

several previously unknown small elements extremely high conserved between mouse and human. Such elements were found around three of the six alternatively included exons (Table 2). The comparison of the conserved intronic sequences around NF1 exons 9br, $10 \mathrm{a}-2,23 \mathrm{a}, 23 \mathrm{~b}$ and $48 \mathrm{a}$ with each other revealed no common motifs.

Taken together, we assume that the few identified conserved elements cannot account for the extent of the high intron conservation. Therefore, we also performed in silico search for evidence indicating the presence of other genes in the conserved intron regions. However, in silico 
search for RNA polymerase II promoter sequences correlating with the conserved regions was inconclusive as different algorithms yielded different putative promoter locations or none at all. Also, no RNA polymerase III promoter sequences and open reading frames in sense and antisense orientation were identified and no matches to elements characteristic for snoRNAs were found.

\section{Discussion}

We describe here a high identity between human and mouse of intron sequences surrounding most alternatively included coding exons of NF1 and four other genes investigated, but not the average of the constitutive exons. Our finding raises the question if this conservation is a general phenomenon. High intron conservation is described around the alternatively included exons Ve of CDK2 and 5 a of Pax6, ${ }^{41,42}$ but the average conservation of the introns surrounding the constitutive exons of the respective genes was not determined. The complete sequencing of human and mouse genomes greatly facilitated comparisons of whole genes. However, the number of homologous genes of human and mouse with conserved alternatively included exons is restricted, because genomewide investigation showed that the alternative exon inclusion comprises only approximately $15 \%$ of all alternative splicing events in contrast to exon skipping, which amounts to $48 \%$ approximately. ${ }^{4}$ In addition, there are often inconsistent data concerning the classification of alternative cassette exons as skipped or included, for example, present or absent in the predominant transcript. Several genome-wide comparisons of expressed sequence tags or full-length cDNA clones with the genomic draft of human and mouse have been carried out to investigate the extent of alternative splicing in the respective genome. ${ }^{43,44}$ It suggests itself to use these databases for a genomic comparison of alternatively spliced genes of human and mouse. ${ }^{7,45}$ Such detailed comparisons will shed more light on the difference between the alternative splice forms and elucidate to what extent our finding can be generalized or if it is specific for the NF1 and the other investigated genes. Our observation of highly conserved intron sequences around alternatively included exons in NF1 and other genes raises the question of the reason for this conservation. The idea of a regulatory function in the expression of the respective exon suggests itself. Alternatively included exons have been reported to be smaller than the average. ${ }^{5}$ This could mean that they need more help from accessory splicing factors and therefore more respective binding elements, in exon or intron, to ensure accurate splicing. So, first, there could be an accumulation of intronic splice elements. We found several highly conserved small elements, some of which also possess sequences of known intronic splice enhancer/silencer elements. But, we did not find a general correlation between known splice elements and the conserved intron sequences. Second, regulation of splicing may be influenced by the secondary structure of the pre-mRNA as found in the rat CGRP exon $4 .{ }^{46}$ This possibility will have to be clarified experimentally. Third, the expression of genes transcribed in antisense orientation may regulate alternative exon inclusion. Such a regulation has been described in vivo for the FGF- $2^{47}$ and HFE genes. ${ }^{48}$ We could identify some putative promoter elements correlating with the conserved regions in silico. But, since the reliability of the available prediction algorithms is not very high, this possibility remains to be tested experimentally. In addition, it is conceivable that yet unknown genes are lying in sense orientation in the regions of high identity. But in spite of a thorough investigation, we did not find an indication for this. Our finding may also be relevant for mutation screening in disease-causing genes with alternatively included exons as NF1. The present screening methods often do not find all mutations in NF1. A reason for this may be that some mutations lie in such conserved intronic regions, which are often not investigated in detail. In summary, we found differences in the conservation between introns around alternatively included and introns around alternatively skipped and constitutive exons in the NF1 and four other genes. This could point to a difference in the regulation of exon inclusion and the regulation of constitutive splicing and alternative exon skipping.

\section{Note}

During the submission of this article, Modrek et al (Nat Genet 2003; 34: 177-180) found by a genome-wide scan a difference in conservation of the sequences of alternatively included exons and constitutive or skipped exons between human and mosue or rat.

\section{Acknowledgements}

We thank $D$ Viskochil for critical discussion concerning our observations. The technical assistance of A Siegel, B Dieske, H Goetz and E Winkler is also gratefully acknowledged. Furthermore, we thank $H$ Lattke, who died recently and $R$ Mueller for technical advice. This work was supported by the Deutsche Krebshilfe $(B B, B D)$ and Graduate College 460 of University Ulm (OK).

\section{References}

1 Zhang MQ: Computational prediction of eukaryotic proteincoding genes. Nat Rev Genet 2002; 3: 698-709.

2 Cartegni L, Chew SL, Krainer AR: Listening to silence and understanding nonsense: exonic mutations that affect splicing. Nat Rev Genet 2002; 3: 285-298.

3 Ladd AN, Cooper TA: Finding signals that regulates alternative splicing in the postgenomic era. Genome Biol 2002; 3: (reviews0008.1-0008.16.). 
4 Nogués G, Kadener S, Cramer P, Bentley D, Kornblihtt AR: Transcriptional activators differ in their abilities to control alternative splicing. J Biol Chem 2002; 277: 43110-43114.

5 Clark F, Thanaraj TA: Categorization and characterization of transcript-confirmed constitutively and alternatively spliced introns and exons from human. Hum Mol Genet 2002; 11: $451-464$

6 Loots GG, Locksley RM, Blankespoor CM et al: Identification of a coordinate regulator of interleukins 4,13 , and 5 by cross-species sequence comparisons. Science 2000; 288: 136-140.

7 Mouse Genome Sequencing Consortium: Initial sequencing and comparative analysis of the mouse genome. Nature 2002; 420: $520-562$.

8 Shen $\mathrm{MH}$, Harper PS, Upadhyaya M: Molecular genetics of neurofibromatosis type 1 (NF1). J Med Genet 1996; 33: 2-17.

9 Cichowski K, Jacks T: NF1 tumor suppressor gene function: narrowing the GAP. Cell 2001; 104: 593-604.

10 Kaufmann D, Muller R, Kenner O et al: The N-terminal splice product NF1-10a-2 of the NF1 gene codes for a transmembrane segment. Biochem Biophys Res Commun 2002; 294: 496-503.

11 Mantani A, Wakasugi S, Yokota Y, Abe K, Ushio Y, Yamamura K: A novel isoform of the neurofibromatosis type-1 mRNA and a switch of isoforms during murine cell differentiation and proliferation. Gene 1994; 148: 245-251.

12 Vandenbroucke I, Vandesompele J, De Paepe A, Messiaen L: Quantification of NF1 transcripts reveals novel highly expressed splice variants. FEBS Lett 2002; 522: 71-76.

13 Wimmer K, Eckart M, Rehder H, Fonatsch C: Illegitimate splicing of the NF1 gene in healthy individuals mimics mutation-induced splicing alterations in NF1 patients. Hum Genet 2000; 106: 311-313.

14 Kaufmann D, Leistner W, Kruse P et al: Aberrant splicing in several human tumors in the tumor suppressor genes neurofibromatosis type 1 , neurofibromatosis type 2 , and tuberous sclerosis 2. Cancer Res 2002; 62: 1503-1509.

15 Thomson SA, Wallace MR: 2002) RT-PCR splicing analysis of the NF1 open reading frame. Hum Genet 2002; 110: 495-502.

16 Jensen KB, Dredge BK, Stefani G et al: Nova-1 regulates neuronspecific alternative splicing and is essential for neuronal viability. Neuron 2000; 25: 359-371.

17 Sirand-Pugnet P, Durosay P, Brody E, Marie J: An intronic (A/ U)GGG repeat enhances the splicing of an alternative intron of the chicken beta-tropomyosin pre-mRNA. Nucleic Acids Res 1995; 23: 3501-3507.

18 Chou MY, Underwood JG, Nikolic J, Luu MH, Black DL: Multisite RNA binding and release of polypyrimidine tract binding protein during the regulation of c-src neural-specific splicing. Mol Cell 2000; 5: 949-957.

19 Carlo T, Sierra R, Berget SM: A 5' splice site-proximal enhancer binds SF1 and activates exon bridging of a microexon. Mol Cell Biol 2000; 20: 3988-3995.

20 Modafferi EF, Black DL: A complex intronic splicing enhancer from the c-src pre-mRNA activates inclusion of a heterologous exon. Mol Cell Biol 1997; 17: 6537-6545.

21 Chabot B, Blanchette M, Lapierre I, La Branche H: An intron element modulating $5^{\prime}$ splice site selection in the hnRNP A1 pre-mRNA interacts with hnRNP A1. Mol Cell Biol 1997; 17: 1776-1786.

22 Jin W, Bi W, Huang ESC, Cote GJ: Glioblastoma cell-specific expression of fibroblast growth factor receptor-1 $\beta$ requires an intronic repressor of RNA splicing. Cancer Res 1999; 59: $316-319$

23 Pret AM, Balvay L, Fiszman MY: Regulated splicing of an alternative exon of beta-tropomyosin pre-mRNAs in myogenic cells depends on the strength of pyrimidine-rich intronic enhancer elements. DNA Cell Biol 1999; 18: 671-683.

24 Blanchette $M$, Chabot B: Modulation of exon skipping by high-affinity hnRNP A1-binding sites and by intron elements that repress splice site utilization. EMBO J 1999; 18: 1939-1952.
25 del Gatto F, Plet A, Gesnel MC, Fort C, Breathnach R: Multiple interdependent sequence elements control splicing of a fibroblast growth factor receptor 2 alternative exon. Mol Cell Biol 1997; 17: $5106-5116$.

26 Lou H, Yang Y, Cote GJ, Berget SM, Gagel RF: An intron enhancer containing a $5^{\prime}$ splice site sequence in the human calcitonin/ calcitonin gene-related paptide gene. Mol Cell Biol 1995; 15: $7135-7142$.

27 Markovtsov V, Nikolic JM, Goldman JA, Turck CW, Chou MY, Black DL: Cooperative assembly of an hnRNP complex induced by a tissue-specific homolog of polypyrimidine tract binding protein. Mol Cell Biol 2000; 20: 7463-7479.

28 Standiford DM, Sun WT, Davis MB, Emerson Jr CP: Positive and negative intronic regulatory elements control muscle-specific alternative exon splicing of drosophila myosin heavy chain transcripts. Genetics 2001; 157: 259-271.

29 Cooper TA: Muscle-specific splicing of a heterologous exon mediated by a single muscle-specific splicing enhancer from the cardiac troponin T gene. Mol Cell Biol 1998; 18: 4519-4525.

30 Zhang MQ: Identification of human gene core promoters in silico. Genome Res 1998; 8: 319-326.

31 Knudsen S: Promoter2.0: for the recognition of Pol II promoter sequences. Bioinformatics 1999; 15: 356-361.

32 Boyd DC, Turner PC, Watkins NJ, Gerster T, Murphy S: Functional redundancy of promoter elements ensures efficient transcription of the human 7SK gene in vivo. J Mol Biol 1995; 253: $677-690$.

33 Weinstein LB, Steitz JA: Guided tours: from precursor snoRNA to functional snoRNA. Curr Opin Cell Biol 1999; 11: $378-384$.

34 Hedges SB, Kumar S: Vertebrate genomes compared. Science 2002; 297: $1283-1285$

35 Melo CA, Serra C, Stoyanova V et al: Alternative splicing of a previously unidentified CFTR exon induces an in-frame stop codon $5^{\prime}$ of the $\mathrm{R}$ region. FEBS Lett 1993; 329: $159-162$.

36 Zylka MJ, Shearman LP, Weaver DR, Reppert SM: Three period homologs in mammals: differential light responses in the suprachiasmatic circadian clock and oscillating transcripts outside of brain. Neuron 1998; 20: 1103-1110.

37 Kim JE, Kim KH, Lee SW, Seol W, Shiba K, Kim S: An elongation factor-associating domain is inserted into human cysteinyl-tRNA synthetase by alternative splicing. Nucleic Acids Res 2000; 28: $2866-2872$.

38 Fukuda M, Ogata Y, Saegusa C, Kanno E, Mikoshiba K: Alternative splicing isoforms of synaptotagmin VII in the mouse, rat and human. Biochem J 2002; 365: 173-180.

39 Hull J, Shackleton S, Harris A: Analysis of mutations and alternative splicing patterns in the CFTR gene using mRNA derived from nasal epithelial cells. Hum Mol Genet 1994; 3: $1141-1146$.

40 Haber DA, Sohn RL, Buckler AJ, Pelletier J, Call MK, Housman DE: Alternative splicing and genomic structure of the Wilms tumor gene WT1. Proc Natl Acad Sci USA 1991; 88: 9618-9622.

41 Ellenrieder C, Bartosch B, Lee GY et al: The long form of CDK2 arises via alternative splicing and forms an active protein kinase with cyclins A and E. DNA and Cell Biol 2001; 20: 413-423.

42 Jaworski C, Sperbeck S, Graham C, Wistow G: Alternative splicing of Pax6 in bovine eye and evolutionary conservation of intron sequences. Biochem Biophys Res Commun 1997; 240: 196-202.

43 Modrek B, Resch A, Grasso C, Lee C: Genome-wide detection of alternative splicing in expressed sequences of human genes. Nucleic Acids Res 2001; 29: 2850-2859.

44 Zavolan M, Van Nimwegen E, Gaasterland T: Splice variation in mouse full-length cDNAs identified by mapping to the mouse genome. Genome Res 2002; 12: 1377-1385.

45 Xuan Z, Wang J, Zhang MQ: Computational comparison of two mouse draft genomes and the human golden path. Genome Biol 2002; 4: R1. 
46 Coleman TP, Roesser JR: RNA secondary structure: an important cis-element in rat calcitonin/CGRP pre-messenger RNA splicing. Biochemistry 1998; 37: 15941-15950.

47 Li AW, Murphy PR: Expression of alternatively spliced FGF-2 antisense RNA transcripts in the central nervous system: regulation of FGF-2 mRNA translation. Mol Cell Endocrinol 2000; 170: $233-242$.
48 Thenie AC, Gicquel IM, Hardy S et al: Identification of an endogenous RNA transcribed from the antisense strand of the HFE gene. Hum Mol Gen 2001; 10: 1859-1866.

49 Rettenberger G, Klett C, Zechner U et al: ZOO-FISH analysis: cat and human karyotypes closely resemble the putative ancestral mammalian karyotype. Chromosome Res 1995; 13: 479-486. 